\title{
Plasma Adropin as a Potential Marker Predicting Obesity and Obesity-associated Cancer in Korean Patients With Type 2 Diabetes Mellitus
}

\author{
Ha-Neul Choi, Jung-Eun Yim \\ Department of Food and Nutrition, College of Natural Science, Changwon National University, Changwon, Korea
}

\begin{abstract}
Background: Type 2 diabetes mellitus (T2DM) and cancer are serious health problems worldwide, and their prevalences have been on the rise in recent years. It has been reported that adropin plays an important role in the development of T2DM, oxidative stress, inflammation, and obesity. However, there is limited information available on T2DM from human studies, especially for the Korean population. In this study, we aimed to investigate the correlation between adropin levels and obesity of Korean T2DM patients.

Methods: Thirty-six T2DM patients were recruited for this study. The participants were further classified into female $(n=12)$ and male $(n=24)$. Their body composition, metabolic parameters, inflammatory factors, and oxidative stress were measured.

Results: The severity of obesity is more manifested in male than in female. Plasma triglyceride (TG) and high-sensitivity C-reactive protein (hs-CRP) levels of male were significantly higher than female. The plasma adropin and adiponectin level of female was significantly higher than male. The body weight, body mass index (BMI), body fat mass were negatively correlated with the plasma adropin level in female, whereas adropin has positive correlation with adiponectin in female. The hs-CRP was negatively correlated with the plasma adropin level in female and male. malondialdehyde, reactive oxidative species, and TNF- $\alpha$ was not significantly correlated with adropin in patients with T2DM.

Conclusions: These findings suggest that adropin may be more used as a biomarker for predicting the risk of obesity and inflammation in Korean patients with T2DM, especially women.
\end{abstract}

(J Cancer Prev 2018;23:191-196)

Key Words: Diabetes mellitus, Obesity, Adropin, Inflammation, Oxidative stress

\section{INTRODUCTION}

Worldwide, diabetes mellitus (DM) and cancer are serious health problem. There is many evidence suggests that DM is associated with increased risk for many forms of cancer. ${ }^{1}$ DM and cancer share many risk factors, such as obesity, inflammatory factors, and oxidative stress.

DM is defined as a chronic disease that is caused either due to i) insufficient production and secretion of insulin and/or ii) a decline in the effectiveness of insulin resulting from inadequate insulin sensitivity in the liver, skeletal muscle, and adipose tissue. ${ }^{2}$ Type 2 diabetes mellitus (T2DM) is generally linked to obesity and insulin resistance and occurs when the cells lose their normal response to the insulin made by the pancreatic $\beta$-cells. ${ }^{3}$ Impaired insulin sensitivity is the major underlying pathology of T2DM, the predominant form of $\mathrm{DM}{ }^{4} \mathrm{DM}$ also leads to low high-density lipoprotein cholesterol (HDL-C), hypertriglyceridemia, and increased oxidative stress-induced endothelial dysfunction. $^{5.6}$ If left unattended, DM can cause severe glucotoxicity and diabetic complications. Low levels of HDL-C, often associated with elevated triglyceride (TG) levels, is the most common pattern of dyslipidemia in individuals with T2DM. ${ }^{5}$

According to the World Health Organization, the global prevalence of DM among adults over 18 years of age has risen from $4.7 \%$ to $8.5 \%$ between the years 1980 to 2014 . $^{7}$ Over the past decade, DM prevalence has escalated more rapidly in the middle-

Received November 19, 2018, Revised December 17, 2018, Accepted December 18, 2018

Correspondence to: Jung-Eun Yim

Department of Food and Nutrition, College of Natural Science, Changwon National University, 20 Changwondaehak-ro, Uichang-gu, Changwon 51140, Korea Tel: +82-55-213-3517, Fax: +82-55-281-7480, E-mail: jeyim@changwon.ac.kr, ORCID: Jung-Eun Yim, https://orcid.org/0000-0001-8344-1386

Copyright (C) 2018 Korean Society of Cancer Prevention

(c) This is an Open Access article distributed under the terms of the Creative Commons Attribution Non-Commercial License (http://creativecommons.org/licenses/by-nc/4.0) which permits unrestricted non-commercial use, distribution, and reproduction in any medium, provided the original work is properly cited. 
and low-income countries than the high-income countries. ${ }^{7}$ In Korea, the prevalence of DM in people $>30$ years of age has increased from $8.9 \%$ (2005) to $11.1 \%$ (2013-2015). ${ }^{8}$ Overall, the incidence and prevalence of T2DM have grown at epidemic levels, resulting in a serious public health problem. ${ }^{3,6,7}$

Uncontrolled DM can develop into cancer due to hyperinsulinemia and hyperglycemia. ${ }^{9-11}$ Excess insulin effected leading to increases in bioavailable sex hormone. Hyperinsulinemia caused increasing androgen in ovaries of premenopausal women. Elevated sex steroids are associated with the high incidence of postmenopausal breast and endometrial cancers. ${ }^{11}$ Also, cancer patients with T2DM or hyperglycemia, the proportion of tumor recurrence ratio and metastasis is higher than in T2DM patients without metabolic disease. ${ }^{10}$ Thus, controlling hyperglycemia and hyperinsulinemia may have important therapeutic implications in DM and DM with cancer patients.

Recent studies have suggested that several peptide hormones play important roles in the modulation of systemic metabolism and energy homeostasis. ${ }^{12,13}$ Adropin, originally identified in $2008,{ }^{13}$ is a product of the Energy Homeostasis Associated (Enho) gene and a peptide hormone containing 72 amino acids, and it is known to play a crucial role in the regulation of energy homeostasis, systemic metabolism, and endothelial function. ${ }^{14-16}$ Adropin-knockout mice have shown severe insulin resistance, dyslipidemia, and the presence of lipid droplets in the liver. ${ }^{17}$ Administration of adropin resulted in improved glucose utilization, hyperinsulinemia, and hepatic steatosis in dietinduced obesity mice and streptozotocin-induced diabetic rats. ${ }^{18,19}$ In addition, chronic administration of adropin reduced monocyte/ macrophage infiltrating the intra-plaque and smooth muscle cell content; this consequently alleviated the advancement of atherosclerosis in the aorta of the apolipoprotein E knockout mice. ${ }^{20}$

Low adropin levels are closely related to insulin resistance, dyslipidemia, hepatic steatosis, and increased body fat mass (BFM). Clinical trial studies have revealed that conditions such as T2DM, gestational DM, obesity, metabolic syndrome, non-alcoholic fatty liver disease, polycystic ovary syndrome, coronary atherosclerosis, and central nervous system disorders are also associated with low adropin levels. ${ }^{16,21-25}$ Adropin levels were also determined lower in patients with endometrium cancer than normal group. ${ }^{26}$

However, the relationship between adropin, obesity, DM, and cancer risks has not been well studied in Korean subjects. Therefore, this study was designed and aimed to determine the association between adropin levels, obesity, inflammatory factors, and oxidative stress in Korean patients in T2DM.

\section{MATERIALS AND METHODS}

\section{Subjects and experimental design}

The clinical trial was conducted from April through November 2017. This study was approved by the Institutional Review Board of Changwon Fatima Hospital (IRB No. 17-04). The informed consent was obtained from all participants. Thirty-six T2DM patients were enrolled and classified as female and male. Potential subjects meeting any of these following criteria were excluded from this study: hypertension, cardiovascular diseases (CVDs), kidney disease, depressive disorder, medical or surgical illness (within 6 months of enrollment), and intake of dietary restricted drugs or participation in diet programs (within 3 months of enrollment). All participants visited the hospital at least twice during the entire study period.

\section{Anthropometric measurement}

For all study participants, anthropometric data and body composition were measured at their first visit to the hospital. For each participant, weight and height were measured, and body mass index (BMI) was calculated as weight (kg) divided by height squared $\left(\mathrm{m}^{2}\right)$. BFM and percent BFM were measured using bioimpedance analysis (InBody 3.0; Biospace, Seoul, Korea). Waist circumference (WC) and hip circumference $(\mathrm{HC})$ were measured twice for each participant. The waist and hip circumference ratio was calculated as the WC divided by the HC.

\section{Biochemical analysis}

At the end of the clinical trial, blood samples were collected after a 10-hour overnight fast and centrifuged at $1,500 \times g$ for 15 minutes to separate the plasma. Plasma samples were stored at $-80^{\circ} \mathrm{C}$ until laboratory analysis. The analysis of glucose, hemoglobin A1C (HbAlc), total cholesterol (TC), TG, HDL-C, and low-density lipoprotein cholesterol (LDL-C) were measured by enzymatic methods using commercial kits (Asan Pharmaceutical Co., Seoul, Korea).

4. Analysis of adropin, inflammatory factors, and oxidative stress

Plasma adropin protein (Elabscience Biotechnology Co., Wuhan, China), adiponectin (ALPCO, Keewaydin Drive, Salem, $\mathrm{NH}$, USA) and high-sensitivity C-reactive protein (hs-CRP) (BioVendor Laboratorní Medicina a.s, Modrice, the Czech Republic) were measured by human-specific enzyme-linked immunosorbent assay kits. TNF- $\alpha$ and malondialdehyde (MDA) were determined using ELISA kits from Enzo Life Sciences, Inc. 
(Farmingdale, NY, USA). Reactive oxidative species (ROS) was determined using OxiSelect Intracellular ROS Assay Kit (Cell Biolabs Inc., San Diego, CA, USA).

\section{Statistical analysis}

Statistical analyses were performed with IBM SPSS software ver. 24.0 (IBM Co., Armonk, NY, USA). Data were expressed as the mean $\pm \mathrm{SD}$, and the value of $P<0.05$ was regarded as statistically significant. For the participants, the difference between female and male was analyzed using $t$-test.

\section{RESULTS}

\section{Anthropometric data}

General characteristics and body compositions of the subjects are shown in Table 1. Mean age of female and male were $48.8 \pm$

Table 1. Age and anthropometric measurements in patients with T2DM

\begin{tabular}{lccc}
\hline \multicolumn{1}{c}{ Variable } & $\begin{array}{c}\text { Female } \\
(\mathrm{n}=12)\end{array}$ & $\begin{array}{c}\text { Male } \\
(\mathrm{n}=24)\end{array}$ & $P$-value \\
\hline Age $(\mathrm{yr})$ & $48.8 \pm 8.8$ & $42.8 \pm 10.7$ & 0.107 \\
Weight $(\mathrm{kg})$ & $60.3 \pm 10.2$ & $79.6 \pm 18.2$ & 0.002 \\
Height $(\mathrm{cm})$ & $159.7 \pm 4.5$ & $171.4 \pm 6.1$ & 0.000 \\
BMI $\left(\mathrm{kg} / \mathrm{m}^{2}\right)$ & $23.6 \pm 3.9$ & $27.0 \pm 5.4$ & 0.063 \\
BFM $(\mathrm{kg})$ & $19.3 \pm 6.1$ & $22.8 \pm 12.7$ & 0.373 \\
PBFM $(\%)$ & $31.4 \pm 4.9$ & $27.2 \pm 8.0$ & 0.100 \\
WC $(\mathrm{cm})$ & $80.6 \pm 12.2$ & $91.8 \pm 12.0$ & 0.012 \\
HC $(\mathrm{cm})$ & $89.8 \pm 8.8$ & $99.2 \pm 9.8$ & 0.008 \\
WHR & $0.9 \pm 0.1$ & $0.9 \pm 0.1$ & 0.491 \\
\hline
\end{tabular}

Values are presented as mean $\pm \mathrm{SD}$. $P$-values between genders were statistically analyzed by $t$-test. T2DM, type 2 diabetes mellitus; BMI, body mass index; BFM, body fat mass; PBFM, percent body fat mass; WC, waist circumference; HC, hip circumference; WHR, waist and hip circumference ratio.

Table 2. Glycemic and lipid profiles in patients with T2DM

\begin{tabular}{|c|c|c|c|}
\hline Variable & Female & Male & $P$-value \\
\hline Glucose $(\mathrm{mg} / \mathrm{dL})$ & $122.8 \pm 33.5$ & $129.0 \pm 30.3$ & 0.576 \\
\hline HbAlc (\%) & $7.8 \pm 1.6$ & $6.3 \pm 3.4$ & 0.190 \\
\hline TC (mg/dL) & $183.6 \pm 43.8$ & $174.1 \pm 30.7$ & 0.454 \\
\hline TG (mg/dL) & $110.5 \pm 63.9$ & $188.5 \pm 115.5$ & 0.037 \\
\hline HDL-C (mg/dL) & $32.2 \pm 12.2$ & $32.7 \pm 9.1$ & 0.906 \\
\hline LDL-C (mg/dL) & $59.8 \pm 16.4$ & $54.6 \pm 11.0$ & 0.269 \\
\hline Atherogenic index & $5.3 \pm 1.9$ & $4.6 \pm 1.4$ & 0.251 \\
\hline
\end{tabular}

Values are presented as mean $\pm \mathrm{SD}$. $P$-values between genders were statistically analyzed by $t$-test. T2DM, type 2 diabetes mellitus HbAlc, hemoglobin A1C (blood glycated haemoglobin); TC, total cholesterol; TG, triglyceride; HDL-C, high-density lipoprotein cholesterol; LDL-C, low-density lipoprotein cholesterol.
8.8 and $42.8 \pm 10.7$ years, respectively. Compared with female, body weight, WC, and HC of male were significantly higher ( $P=$ $0.002,0.012$, and 0.008 , respectively). Between the two genders, female did not show abdominal obesity $(80.6 \pm 12.2 \mathrm{~cm})$, whereas abdominal obesity were found in male $(91.8 \pm 12.0 \mathrm{~cm})$. This observation suggested that degree of obesity is more severely manifested in male than in female.

\section{Glycemic and lipid profiles}

Blood levels of glycemic indices and lipid profiles are listed in Table 2. The levels of glucose, HbA1c, TC, HDL-C, LDL-C, and atherogenic index did not show any differences in both genders. Plasma TG level was significantly elevated in male than female $(P=0.037)$.

\section{Adropin, inflammatory factors, and oxidative stress}

Plasma adropin, cytokines, and oxidative stress of the subjects are shown in Table 3. Relative to male, adropin and adiponectin were significantly increased in female ( $P=0.004$ and 0.013$)$. The MDA and ROS of the male group tended to increase, compared to female group. However, no significant difference was observed. Plasma hs-CRP levels of male were significantly higher than female $(P=0.017)$.

\section{Correlation of plasma adropin levels and obesity}

We evaluated the age-adjusted correlation between the plasma levels of adropin and body composition in our study data (Table 4). The association of adropin levels and variable indicators had gender differences existed in participants. In female, weight, BMI, WC, and BFM were negatively correlated with the plasma adropin levels ( $P=0.011,0.036$, and 0.019, respectively). The body composition was not significantly correlated with serum adropin levels in male.

Table 3. Adropin, inflammatory factors, and oxidative stress in patients with T2DM

\begin{tabular}{lccc}
\hline \multicolumn{1}{c}{ Variable } & Female & Male & $P$-value \\
\hline Adropin $(\mathrm{ng} / \mathrm{mL})$ & $0.7 \pm 0.3$ & $0.4 \pm 0.2$ & 0.004 \\
Adiponectin $(\mu \mathrm{g} / \mathrm{mL})$ & $2.9 \pm 1.5$ & $1.6 \pm 0.9$ & 0.013 \\
MDA $(\mu \mathrm{M})$ & $6.5 \pm 2.7$ & $9.1 \pm 5.3$ & 0.065 \\
ROS $(\mathrm{IU} / \mathrm{mL})$ & $62.9 \pm 34.2$ & $84.4 \pm 124.8$ & 0.564 \\
hs-CRP $(\mu \mathrm{g} / \mathrm{mL})$ & $0.5 \pm 0.6$ & $2.6 \pm 3.9$ & 0.017 \\
TNF- $\alpha(\mathrm{pg} / \mathrm{mL})$ & $23.8 \pm 30.1$ & $15.3 \pm 2.2$ & 0.351 \\
\hline
\end{tabular}

Values are presented as mean $\pm \mathrm{SD}$. $P$-values between genders were statistically analyzed by $t$-test. T2DM, type 2 diabetes mellitus; MDA, malondialdehyde; ROS, reactive oxygen species; hs-CRP, high-sensitivity C-reactive protein. 
Table 4. Age-adjusted correlation coefficients $(r)$ of adropin and body composition in patientswith T2DM

\begin{tabular}{llr}
\hline Variable & Female & Male \\
\hline Weight & $-0.757^{* *}$ & -0.242 \\
BMI & $-0.727^{*}$ & -0.226 \\
Waist & $-0.635^{*}$ & -0.043 \\
Hip & -0.485 & -0.256 \\
WHR & -0.287 & 0.314 \\
BFM & $-0.688^{*}$ & -0.212 \\
\hline
\end{tabular}

T2DM, type 2 diabetes mellitus; BMI, body mass index; WHR, waist and hip circumference ratio; BFM, body fat mass. $* P<0.05$ and $* * P<0.01$.

\section{Correlation of plasma adropin, inflammatory factors, and oxidative stress}

We presented the age-adjusted correlation between the plasma levels of adropin and inflammatory factors, and oxidative stress in our study data (Table 5). The hs-CRP a significant negative association with the adropin levels in female and male groups ( $P=$ 0.008 and 0.013 , respectively). The adiponectin level demonstrated a positive correlation with the adropin in female $(P=0.001)$. MDA, ROS, and TNF- $\alpha$ was not significantly correlated with adropin in male and female.

\section{DISCUSSION}

We investigated an association between variable parameters and adropin levels in a group of Korean T2DM patients. The anthropometric parameters, metabolic profiles, and cancer risks were measured for the participating patients.

In this study, all participants were taking oral hypoglycemic agents. Therefore, we observed no significant differences between the both genders in their glycemic indices, including fasting blood glucose and HbAlc. Plasma TG level of female was significantly lower than male. TC, LDL-C, and atherogenic index of female appeared to be higher than male. These results suggested that T2DM patients can develop a blood lipid disorder with or without obesity in a gender-independent manner. Increased TG and decreased HDL levels can raise the concentrations of remnant cholesterol and lipoproteins, apolipoprotein B, and small dense LDL-C. ${ }^{27}$ Excess LDL-C can cause atherogenic dyslipidemia, which becomes CVDs. Thus, blood lipid parameters such as TG, TC, HDL-C, and LDL-C may serve as reliable predictors of cardiovascular complications. ${ }^{27,28}$ Therefore, both genders with the T2DM require more careful management of their blood lipid profiles to prevent CVDs linked
Table 5. Age-adjusted correlation coefficients $(r)$ of adropin, cytokines, and oxidative stress in patients with T2DM

\begin{tabular}{lcc}
\hline \multicolumn{1}{c}{ Variable } & Female & Male \\
\hline Adiponectin & $0.837^{* *}$ & -0.131 \\
MDA & -0.126 & 0.069 \\
ROS & 0.175 & -0.094 \\
hs-CRP & $-0.746^{* *}$ & $-0.509 *$ \\
TNF- $\alpha$ & -0.419 & 0.485 \\
\hline
\end{tabular}

MDA, malondialdehyde; ROS, reactive oxygen species; hs-CRP, high-sensitivity C-reactive protein. ${ }^{*} P<0.05$ and ${ }^{*} P<0.01$.

to DM.

Adropin levels were significantly higher in female than male, whereas female displayed low hs-CRP concentrations than male. With an increase in adropin level, body fat indices and plasma TG appeared to decrease significantly in women. In addition, there was a significant negative correlation between hs-CRP and adropin levels in both two genders.

Adropin is a peptide hormone that is found in the liver, pancreas, kidney, heart, and brain. ${ }^{29}$ It is known to play roles in metabolic homeostasis including energy regulation and macronutrient. ${ }^{17,18}$ Many studies have reported that serum adropin levels decrease in T2DM and obese patients. ${ }^{17.30}$ Adropin regulates glucose oxidation by activating pyruvate dehydrogenase in muscle. ${ }^{31}$ In the current study, we did not find a correlation of plasma adropin with the glycemic profile including fasting blood glucose and HbAlc. These observations might have resulted due to the regular use of a hypoglycemic reagent for a long-term by the participants. Additionally, adropin plays a role in the modulation of lipid homeostasis by regulating the expression of hepatic transcription factors. ${ }^{18}$ It is involved in the liver $\mathrm{X}$ receptors $\alpha$ (LXR $\alpha$ ) regulation which promotes the synthesis and storage of TG in liver and blood. ${ }^{18,32}$ Thus, low adropin concentration does not control the LXR $\alpha$ expression, resulting in the elevation of TG level. Adropin also regulates angiogenesis via upregulation of endothelial nitric oxide synthase expression, suggesting that it may be critical for maintaining vascular function and preventing CVDs. ${ }^{14,24}$ Further, adropin regulates lipid oxidation via inhibiting carnitine palmitoyltransferase in muscle. ${ }^{31}$

Excess adipose tissue releases adipocytes-derived hormone modulating lipid and glucose metabolism, inflammation, and atherosclerosis. ${ }^{33}$ Among the various adipokines, adiponectin provide an important relationship between obesity, insulin resistance, T2DM, and related inflammatory disorders. ${ }^{33.34}$ Adiponectin involved in insulin sensitivity and fat oxidation in the liver and muscle. ${ }^{35}$ Decreased adiponectin contribute to insulin resistance associated with obesity. ${ }^{33.35}$ Our study showed 
that plasma adiponectin of female was significantly higher than male. These results thought that certain gender- specific difference. Earlier study reported that androgen (male sex hormone) on the down-regulation of adiponectin production. ${ }^{36}$

Female adropin overexpression transgenic (Adr-Tg) mice exhibited an increase in total serum adiponectin, while serum TG was also reduced in Adr-Tg mice. Also, adropin treatment ameliorated hyperinsulinemia and increased serum adiponectin in diet induced obesity mice. ${ }^{18}$ These observations suggested that adropin ameliorated fat accumulation and also associated with improvement adiponectin production status.

Elevated levels of hs-CRP have been associated with an increased risk of developing T2DM. ${ }^{19}$ Akcilar et al. ${ }^{19}$ reported that adropin possibly inhibits the expression of TNF- $\alpha$ and interleukin-6 (IL-6) of the pancreas in the adropin-treated DM group. Thus, improving of adropin levels may affect inflammation status and prevent cancer. Accumulation of fat mass increases the expression of hs-CRP by IL- 6 stimulation in the liver. ${ }^{19,37.38}$ However, the effects of adropin on inflammation are not yet fully understood.

In conclusion, our study showed a decrease in the adropin levels in male than female. With an increase in the adropin level, a corresponding decrease in fat accumulation, plasma TG and inflammation status was observed. Plasma adropin level correlated positively with plasma adiponectin level in female and with hs-CRP in female and male. Therefore, adropin may be used as a biomarker for predicting the risk of obesity and inflammation in T2DM. However, to gain a better insight and elucidate the relevant mechanisms, further studies need to be conducted to study the association of biomarkers, cancer risks, and T2DM.

\section{ACKNOWLEDGMENTS}

This work was supported by the National Research Foundation of Korea (NRF) grant funded by the Korea government (NRF-2016R1D1A1B03935660).

\section{CONFLICTS OF INTEREST}

No potential conflicts of interest were disclosed.

\section{REFERENCES}

1. Giovannucci E, Harlan DM, Archer MC, Bergenstal RM, Gapstur SM, Habel LA, et al. Diabetes and cancer: a consensus report. CA Cancer J Clin 2010;60:207-21.

2. American Diabetes Association. Classification and diagnosis of diabetes: standards of medical care in diabetes-2018. Diabetes Care 2018;41:S13-27.

3. Eckel RH, Kahn SE, Ferrannini E, Goldfine AB, Nathan DM, Schwartz MW, et al. Obesity and type 2 diabetes: what can be unified and what needs to be individualized? J Clin Endocrinol Metab 2011;96:1654-63.

4. Scheen AJ. Pathophysiology of type 2 diabetes. Acta Clin Belg 2003:58:335-41.

5. O'Keefe JH Jr, Miles JM, Harris WH, Moe RM, McCallister BD. Improving the adverse cardiovascular prognosis of type 2 diabetes. Mayo Clin Proc 1999:74:171-80.

6. Maritim AC, Sanders RA, Watkins JB 3rd. Diabetes, oxidative stress, and antioxidants: a review. J Biochem Mol Toxicol 2003; 17:24-38.

7. World Health Organization. Diabetes. http://www.who.int/newsroom/fact-sheets/detail/diabetes. Accessed November 15, 2017.

8. Lee JW, Kang HT, Lim HJ, Park B. Trends in diabetes prevalence among Korean adults based on Korean National Health and Nutrition Examination Surveys III-VI. Diabetes Res Clin Pract 2018; 138:57-65.

9. Duan W, Shen X, Lei J, Xu Q, Yu Y, Li R, et al. Hyperglycemia, a neglected factor during cancer progression. Biomed Res Int 2014:2014:461917.

10. Calle EE, Kaaks R. Overweight, obesity and cancer: epidemiological evidence and proposed mechanisms. Nat Rev Cancer 2004:4:579-91.

11. Vander Heiden MG, Cantley LC, Thompson CB. Understanding the Warburg effect: the metabolic requirements of cell proliferation. Science 2009:324:1029-33.

12. Barrera JG, Sandoval DA, D'Alessio DA, Seeley RJ. GLP-1 and energy balance: an integrated model of short-term and long-term control. Nat Rev Endocrinol 2011;7:507-16.

13. Field BC, Chaudhri OB, Bloom SR. Bowels control brain: gut hormones and obesity. Nat Rev Endocrinol 2010;6:444-53.

14. Lovren F, Pan Y, Quan A, Singh KK, Shukla PC, Gupta M, et al. Adropin is a novel regulator of endothelial function. Circulation 2010;122:S185-92.

15. Aydin S, Kuloglu T, Aydin S, Eren MN, Yilmaz M, Kalayci M, et al. Expression of adropin in rat brain, cerebellum, kidneys, heart, liver, and pancreas in streptozotocin-induced diabetes. Mol Cell Biochem 2013:380:73-81.

16. Sayın O, Tokgöz Y, Arslan N. Investigation of adropin and leptin levels in pediatric obesity-related nonalcoholic fatty liver disease. J Pediatr Endocrinol Metab 2014;27:479-84.

17. Ganesh Kumar K, Zhang J, Gao S, Rossi J, McGuinness OP, Halem $\mathrm{HH}$, et al. Adropin deficiency is associated with increased adiposity and insulin resistance. Obesity (Silver Spring) 2012;20: 1394-402.

18. Kumar KG, Trevaskis JL, Lam DD, Sutton GM, Koza RA, Chouljenko VN, et al. Identification of adropin as a secreted factor linking dietary macronutrient intake with energy homeostasis and lipid metabolism. Cell Metab 2008;8:468-81.

19. Akcilar R, Kocak FE, Simsek H, Akcilar A, Bayat Z, Ece E, et al. Antidiabetic and hypolipidemic effects of adropinin streoptozotocin-induced type 2 diabetic rats. Bratisl Lek Listy 2016; 117:100-5.

20. Sato K, Yamashita T, Shirai R, Shibata K, Okano T, Yamaguchi M, et al. Adropin contributes to anti-atherosclerosis by suppressing monocyte-endothelial cell adhesion and smooth muscle cell 
proliferation. Int J Mol Sci 2018;19:E1293.

21. Kume T, Calan M, Yilmaz O, Kocabas GU, Yesil P, Temur M, et al. A possible connection between tumor necrosis factor alpha and adropin levels in polycystic ovary syndrome. J Endocrinol Invest 2016;39:747-54

22. Yildirim B, Celik O, Aydin S. Adropin: a key component and potential gatekeeper of metabolic disturbances in policystic ovarian syndrome. Clin Exp Obstet Gynecol 2014:41:310-2.

23. Celik E, Yilmaz E, Celik O, Ulas M, Turkcuoglu I, Karaer A, et al. Maternal and fetal adropin levels in gestational diabetes mellitus. J Perinat Med 2013;41:375-80.

24. Yosaee S, Soltani S, Sekhavati E, Jazayeri S. Adropin- a novel biomarker of heart disease: a systematic review article. Iran J Public Health 2016;45:1568-76.

25. Shahjouei S, Ansari S, Pourmotabbed T, Zand R. Potential roles of adropin in central nervous system: review of current literature. Front Mol Biosci 2016;3:25.

26. Nergiz S, Altinkaya SO, Kurt Ömürlü İ, Yuksel H, Küçük M, Demircan Sezer S. Circulating adropin levels in patients with endometrium cancer. Gynecol Endocrinol 2015:31:730-5.

27. Klop B, Elte JW, Cabezas MC. Dyslipidemia in obesity: mechanisms and potential targets. Nutrients 2013:5:1218-40.

28. Pitha J, Kovár J, Blahová T. Fasting and nonfasting triglycerides in cardiovascular and other diseases. Physiol Res 2015;64 Suppl 3:S323-30.

29. Zang $\mathrm{H}$, Jiang F, Cheng $\mathrm{X}, \mathrm{Xu} \mathrm{H}, \mathrm{Hu} \mathrm{X}$. Serum adropin levels are decreased in Chinese type 2 diabetic patients and negatively correlated with body mass index [published online ahead of print
April 17, 2018]. Endocr J. doi: 10.1507/endocrj.EJ18-0060.

30. Wu L, Fang J, Chen L, Zhao Z, Luo Y, Lin C, et al. Low serum adropin is associated with coronary atherosclerosis in type 2 diabetic and non-diabetic patients. Clin Chem Lab Med 2014;52:751-8.

31. Gao S, McMillan RP, Jacas J, Zhu Q, Li X, Kumar GK, et al. Regulation of substrate oxidation preferences in muscle by the peptide hormone adropin. Diabetes 2014;63:3242-52.

32. Kalaany NY, Mangelsdorf DJ. LXRS and FXR: the yin and yang of cholesterol and fat metabolism. Annu Rev Physiol 2006;68: 159-91.

33. Rabe K, Lehrke M, Parhofer KG, Broedl UC. Adipokines and insulin resistance. Mol Med 2008;14:741-51.

34. Sheng T, Yang K. Adiponectin and its association with insulin resistance and type 2 diabetes. J Genet Genomics 2008;35:321-6.

35. Kadowaki T, Yamauchi T, Kubota N, Hara K, Ueki K, Tobe K. Adiponectin and adiponectin receptors in insulin resistance, diabetes, and the metabolic syndrome. J Clin Invest 2006;116: 1784-92.

36. Nishizawa H, Shimomura I, Kishida K, Maeda N, Kuriyama H, Nagaretani $\mathrm{H}$, et al. Androgens decrease plasma adiponectin, an insulin-senitizing adipocyte-derived protein. Diabetes 2002;51: 2734-41.

37. Weisberg SP, McCann D, Desai M, Rosenbaum M, Leibel RL, Ferrante AW Jr. Obesity is associated with macrophage accumulation in adipose tissue. J Clin Invest 2003;112:1796-808.

38. Lumeng CN, Deyoung SM, Bodzin JL, Saltiel AR. Increased inflammatory properties of adipose tissue macrophages recruited during diet-induced obesity. Diabetes 2007:56:16-23. 